\title{
La difusión tecnológica y la industria aeroespacial en Sonora
}

\author{
Francisco. G. Chávez Martínez ${ }^{1}$
}

\begin{abstract}
Resumen
El artículo presenta una descripción breve de algunas teorías de la integración económica que nos permitirán encontrar los elementos, desde la perspectiva del desarrollo endógeno, la relación de la industria aeroespacial con la economía local. Por tanto, el objetivo del artículo radica en conocer el proceso de difusión tecnológica, la evolución y proyección que presenta la industria aeroespacial en la entidad.
\end{abstract}

Palabras clave: Integración económica, desarrollo endógeno, difusión tecnológica e industria aeroespacial.

Recibido: 25 de noviembre del 2017.

Aceptado: 8 de enero del 2018.

\begin{abstract}
The article presents a brief description of some theories of economic integration that will allow us to find the elements, from the perspective of endogenous development, the ratio of the aerospace industry to the local economy. Therefore, the aim of the article is in the process of technological diffusion, evolution and projection presents the aerospace industry in the state.
\end{abstract}

Keywords: Economic integration, endogenous development, technology diffusion and aerospace.

\footnotetext{
${ }^{1}$ Francisco G. Chávez Martínez. E-mail: fco.chavez.mtz@gmail.com. Estudiante de la Maestría en Integración Económica de la Universidad de Sonora. El presente artículo forma parte del proyecto de tesis, La industria aeroespacial en Sonora: difusión tecnológica $1993-2012$.
} 


\section{Introducción}

Para abordar el proceso de difusión tecnológica, la evolución y proyección que presenta la industria aeroespacial en la entidad, es necesario revisar conceptos básicos que son clave para formar un contexto.

La integración económica es la perspectiva con la cual se interpretan las relaciones entre países que por presiones económicas, políticas y sociales optan por agruparse en bloques económicos que los coloquen en posiciones favorables que permitan la resolución de problemas causados por tendencias económicas globales. Este hecho ha puesto en contacto a regiones que compiten en términos económicos, actualmente los resultados dibujan un panorama muy desigual entre regiones, este panorama abre el debate sobre como contrarrestar la desigualdad. En este sentido, la perspectiva del desarrollo endógeno se propone como medio para contraponer la desigualdad regional que impera particularmente en Latinoamérica.

El desarrollo endógeno es definido como:

La capacidad de una determinada región para generar bienestar colectivo a partir de las condiciones y patrimonio de recursos naturales disponibles, incorporando democráticamente y con protagonismo a los actores locales y correspondientes conocimientos técnicos y culturales (Vázquez Barquero, 2005:3).

Es aquí donde la difusión tecnológica aparece como coadyuvante del proceso de desarrollo endógeno. La integración económica ha permitido a México el contacto con grandes industrias como la aeroespacial. Por sus características tecnológicas la industria aeroespacial es considerada como sector clave en la estrategia para el desarrollo nacional y regional, por representar una fuente de conocimientos y tecnologías que aportan al perfeccionamiento del "saber hacer" local. Existen numerosos trabajos dentro de esta temática de estudio que se concentran en el origen, evolución y proyección de la innovación, sin profundizar en los factores que facilitan la difusión tecnológica.
Para lograr el objetivo el trabajo se divide en un primer apartado sobre los fundamentos teóricos de la integración económica y la difusión tecnológica; un segundo momento del documento aborda a la industria aeroespacial como motor para el desarrollo de la economía mexicana y en especial de Sonora; por último se hace referencia a las conclusiones y la bibliografía citada.

\section{Integración económica y difusión tecnológica: fundamentos teóricos}

La palabra integración proviene del latín; integratio, sin embargo en esta lengua significa renovación. Según el profesor Fritz Machlup (1977) el término integratio es usado en el sentido de combinar las partes en un todo y data de 1600. Sin embargo, el concepto de integración económica tiene una historia bastante corta. Quién finalmente estableció y difundió el término de integración económica fue Jan Tinbergen en su libro Integración económica International (1970) donde hace relación entre integración económica internacional con el libre comercio (Conesa, 1982).

Pero, ¿qué es la integración económica?, es un proceso que se inicia cuando varios países geográficamente cercanos se unen y forman un bloque económico regional sustentado en las preferencias comerciales de los miembros; también hay que considerar que la integración económica es vista como un medio y no un fin, (Conesa, 1982). Es decir, contribuye y es parte del proceso de crecimiento y desarrollo económico de una localidad.

En este apartado se exponen algunas teorías de la integración económica que son elementales para comprender y entender la integración económica entre los países. Las teorías de la integración es un aglomerado de perspectivas científicas, éstas se han complementado unas con otras. Tenemos claro entonces, que para abordar la integración económica debemos tener presente su carácter multidimensional para obtener mejores resultados. 
Teoría económica de las uniones aduaneras: una aproximación al comercio

En 1950 la teoría de la integración económica fue abordada de manera minuciosa por Jacob Viner, según este autor, lo que se busca con una unión aduanera es obtener beneficio, y éste es la diferencia entre creación de comercio y desviación de comercio. La creación de comercio tiene origen en el desplazamiento de la producción, ahí donde los costos de producción son menores; en la desviación de comercio ocurre lo contrario, el desplazamiento de la producción, se va hacia donde los precios son más elevados, antes de la creación de la unión aduanera sus costos eran menores. La creación de comercio se presenta cuando la producción doméstica de un país miembro de una unión aduanera, se sustituye por importaciones de menor costo provenientes de otro país miembro, alcanzando bienestar y especialización. La desviación de comercio se expresa cuando las importaciones de menor costo provenientes de un país fuera de la unión son remplazadas por importaciones de mayor costo del país miembro de la unión, ocurre casi de manera contraria, solo que ahora aparece un país externo a la unión, y de manera contraria, no crea bienestar, la asignación de recursos no es eficiente.

En su trabajo Viner (1950), muestra dentro de ciertos límites y supuestos, la forma en que la unión aduanera opera, así como los efectos positivos y negativos que afectan a los países miembros de una unión aduanera y al resto del mundo. Para Viner, la unión aduanera es un medio para aumentar el mercado nacional y por consecuencia extender los intercambios comerciales. Dado lo anterior, podemos asentar que su estudio solo se enfocó en la producción de bienes y servicios que se intercambiarían en los diferentes mercados miembros de la unión aduanera.

Viner (1950) demostró que las posiciones del pensamiento económico de libre comercio y proteccionistas, no son contradictorias.
Habrá ocasiones donde las decisiones tomadas dentro de la unión aduanera parezcan ser en contra de estas dos corrientes de pensamiento a corto plazo, pero en realidad a largo plazo, las cosas mejoran de manera individual y general.

\section{Teoría de la integración económica: una introducción}

El siguiente aporte significativo respecto a la teorización de la integración económica fue expuesto por Balassa Teoría de la integración económica: una introducción (1961).

En esta obra, Balassa, explica la integración económica como un proceso que se profundiza a medida que sus integrantes o participes de un espacio determinado terminan por aplicar políticas planeadas conjuntamente para obtener beneficios de manera conjunta y que muchas de las veces solo a largo plazo se pueden disfrutar de los beneficios. En muchas ocasiones la espera es demasiada que pone en duda el compromiso.

Entendemos por integración económica como un proceso de acercamiento económico, político y social involucrando a diferentes países. Es posible describir los diferentes niveles y formas de la integración en un esquema propuesto por Balassa: Zona de libre cambio, Unión aduanera, Mercado común, Unión económica y Comunidad económica.

Todas estas etapas, sucesivas y alcanzadas por los países miembros de la Unión Europea, corresponden a las descritas por Balassa.

Como lo menciona Dupuigrenet-Desroussilles Guy, (1964) su obra se divide en tres partes, la primera, examina los efectos de la integración desde un punto de vista estático (repartición de los recursos al interior y al exterior de la unión con un estudio separado de los efectos de la eliminación de barreras comerciales, y la movilidad de los recursos de producción). La segunda parte, estudia los mismos efectos pero ahora de un punto de vista dinámico (relación entre el tamaño de las naciones y su crecimiento económico, problemas de las económicas externas e internas, efectos de integración 
sobre la estructura de mercados, el progreso tecnológico, el comercio exterior, inversión). Y finalmente, Balassa propone instituciones supracionales que permitan la operatividad de estas políticas con el objetivo de lograr la armonización de sus políticas.

François Perroux (1967) ¿Quién integra? ¿En beneficio de quien se realiza la integración? En este trabajo, Perroux, intenta responder a esta pregunta, empezando por analizar y definir el factor que integra a las seis naciones de la pequeña Europa; para Perroux cada Estado nacional tiene diferentes intereses y poderes para impulsar la integración, además, los grupos económicos financieros poseen una fuerza de integración y desintegración que nos indica que la proporción en que se contribuye a la integración es muy desigual entre el grupo de los seis. Perroux (1967:37), cita a Arrow: “...hay que renunciar a la idea de que la competencia es el mejor distribuidor de los recursos”. Lo anterior lo hace con la finalidad de mostrar su desacuerdo con la teoría Walraso-Paretiano del equilibrio de mercado y explicar que la integración económica no es solo economías ligadas por mercancías y precios o extensiones de mercados. Además, para Perroux no se abordan con claridad las desviaciones que puedan presentarse en el equilibrio Walraso-Paretiano. Hay elementos donde el mercado no puede ser el mejor asignado de los recursos y ese espacio o desviación del equilibrio Paretiano exige respuesta para interpretar de mejor manera las diferencias en el análisis de las relaciones entre conjuntos estructurados y sometidos a regímenes económicos diferentes. Ahora bajo el equilibrio Paretiano podemos resumir que es el mercado quien integra y en beneficio de todos se realiza la integración.

Perroux (1967) caracteriza y determina las políticas económicas, para demostrar que las preguntas de ¿quién integra? y ¿en beneficio de quién se realiza la integración? tienen sentido, pues las potencias integradoras pueden lograr provecho de las zonas integradas sin que la reciprocidad sea igualmente verdadera.
Para esto Perroux analiza la integración de varios espacios económicos entre sí. Según Perroux, los efectos de arrastre e impulsión por parte de las industrias son determinantes para comprender y dominar el proceso de crecimiento, pero estos se dan de manera espontánea hacia múltiples direcciones y es aquí donde entra en juego el Estado para mitigar y dirigir de buena manera esos efectos de impulsión y arrastre. Y hace el apunte que países como Francia e Inglaterra implementaron políticas industriales que permitieron sobrellevar de buena manera los efectos de impulsión y arrastre, formando eslabones industriales y una política que permitieron convertir los efectos en crecimiento económico. En el caso anterior, ¿quién integra? ¿en beneficio de quién se realiza la integración?, en cierto grado siempre es el poder público, según las condiciones de la integración y cuando la integración no es aceptada o clara, es cuando el poder público tiene que intervenir para integrar, ahora en respuesta a la segunda cuestión, ¿en beneficio de quién se integra? todo dependerá de la estructura social y de la forma de repartición de la riqueza.

Así pues, la integración por diferentes y numerosos espacios económicos, se presenta de diferente manera y que hay que examinar siempre las estructuras políticas, económicas y sociales existentes para interpretar de mejor manera la integración económica y que la acción por parte de los Estados sea más efectiva.

En los últimos tiempos, y particularmente a partir de la década de 1990 el término tecnología se ha incrustado a nuestro vocabulario de tal manera que es imposible formular ideas sobre crecimiento económico sin tener presente el término tecnológico en el análisis. El avance tecnológico permitió que la comunicación rompiera con los límites establecidos por la distancia. En este sentido facilitó a las empresas mantener cierta presencia de manera simultánea a lo largo del mundo. En el análisis económico la tecnología es reconocida por los autores como factor determinante del crecimiento económico. A sí mismo la tecnología permite a los sistemas productivos ser competitivos y sobre salir en mercados internacionales. 
El concepto difusión permite tener idea de la velocidad con la cual se propagan las innovaciones y las tecnologías. La difusión de las innovaciones y tecnologías se refiere a un proceso que puede ser comparado al de una moda (Crane-Herve, 1969). La visión de la difusión también es planteada (Havelock, 1969), como un proceso de comunicación que tiene la finalidad de informar a utilizadores potenciales de la existencia de una innovación. Se han creado modelos de difusión para la adopción de tecnologías y la difusión de innovaciones, el modelo supone que la adopción de una innovación en la población estará determinada por la rapidez con la cual reacciona el sistema productivo y el entorno socio-institucional. ${ }^{1}$

Para entender el término de difusión aplicado en el desarrollo regional y local, es necesario recurrir a ciertos conceptos para construir el contexto que da lugar al proceso de difusión y de esta manera entender su origen y el papel que juega como variable endógena de crecimiento. Los conceptos de territorio, red, aprendizaje, distrito industrial y entorno innovador. Para Tremblay (2006), el interés en estos conceptos se atribuye a que son pilares importantes para detonar las innovaciones y la capacidad creadora con miras a mejorar la competitividad de las regiones, y es a través de la organización del sistema productivo local, que se construyen estos pilares. Las innovaciones y cambios tecnológicos surgen en un territorio y están asociados al saber hacer local. Tremblay (2006), menciona que el territorio juega un rol importantísimo en la capacidad creadora e innovadora de la región. Es decir, existe una relación entre territorio e innovación y entonces se puede decir, que el desarrollo endógeno tiene un carácter territorial.

La red es definida como: “...el sistema de relaciones y/o contactos que vinculan a las empresas entre sí, cuyo contenido puede referirse a bienes materiales, información y tecnología” (Vázquez Barquero, 1999:98).
Por otro lado, Madhavan la define como “...un conjunto de relaciones cooperativas formalizadas que implican flujos de intercambio de activos, información y estatutos” (2001: 433).

El concepto de red es estratégico para las empresas locales porque sin duda alguna la red se transforma en una herramienta que permite alcanzar ventajas competitivas a través del intercambio de información, conocimiento, tecnología, saber hacer, etc. El concepto de red representa una estructura organizacional con características propias $^{2}$ (Cheriet, 2010).

El aprendizaje es una variable endógena ya que se considera factor determinante del desarrollo regional (Diane-Gabrielle T., 2007). El aprendizaje es la herramienta que permitirá la creación, adopción y asimilación de nuevas tecnologías e innovaciones que revolucionan y organizan el sistema productivo local. Es posible tener acceso al conocimiento a través de compras de licencia o adquisición de patentes, pero el aprendizaje asegura la regeneración de conocimiento (Diane-Gabrielle T., 2007). El aprendizaje no solo transforma la rutina $^{3}$ de las empresas que facultan procesos más eficientes. Para la escuela italiana, el distrito industrial es una red territorializada de pequeñas empresas caracterizadas por la especialización. Según Becattini, (1992:37) el distrito industrial, “Es una entidad socio-territorial caracterizada por la presencia activa de una comunidad de personas y de una población de empresas en un espacio geográfico e histórico determinado [...] tiene a ser una osmosis perfecta entre comunidad local y empresas”. Con esta definición podemos concluir que se trata de una cohesión social condicionada en cierta medida por el elemento de pertenencia expresado en el marco de valores y compromisos que comparten sus habitantes.

El concepto de entorno innovador viene a complementar y ampliar el panorama de distrito industrial respecto las ideas sobre el crecimiento y

\footnotetext{
${ }^{1}$ http://theses.ulaval.ca/archimede/fichiers/21408/ch04.html

${ }^{2}$ La idea de características propias de red es debido que están determinadas también por las características propias del territorio y los elementos que lo componen.

${ }^{3}$ Nelson y Winter, la rutina es análogo al de gen, y las empresas representarían lo que son los organismos vivos en biología.
} 
desarrollo de las empresas locales que organizan su producción.

Esta perspectiva sobre los entornos innovadores fue desarrollada por los investigadores de GREMI ${ }^{1}$ en Suiza. Las ideas propuestas por este grupo de investigadores resaltan la importancia de los aspectos socio-culturales para el desarrollo de entornos innovadores bajo la hipótesis que son los entornos locales quienes generan la innovación. ${ }^{2}$ Lo anterior pone una vez más en la mesa la relación entre territorio e innovación.

El termino de entorno innovador es definido “...como el conjunto localizado de actores que por la frecuencia de sus interacciones o por el simple hecho de vivir en una misma región, comparten un respeto profesional mutuo [...] juntos desarrollan $y$ de manera interdependiente su saber hacer..." (Crevoisier, 1994:36).

La descripción y relación de los conceptos permiten no solo formar una idea sobre cómo se conforman y organizan los sistemas productivos locales. También nos faculta la comprensión del fenómeno de la creación y difusión de las tecnologías e innovaciones. En la actualidad el éxito económico está condicionado por la capacidad creadora e innovadora, y es por esto que se ha hecho hincapié en la importancia de las relaciones e interacciones que tienen las empresas con su entorno, porque sin duda son la base para aumentar la capacidad creadora e innovadora en un espacio determinado. La difusión tecnológica es un medio para la generalización de conocimientos y tecnologías, Según Vázquez Barquero, (1999:133):
La difusión de las innovaciones es un proceso que surge de forma natural, como consecuencia de la aparición de actividades innovadoras. Es una etapa necesaria en el proceso de evolución de la tecnología y su desarrollo está condicionado por la existencia de un entorno socio institucional favorable que lo haga viable.

Es sabido que los investigadores en economía han dedicado su atención en el factor tecnológico por los efectos positivos en la producción. De tal manera el factor tecnológico es situado al centro en el análisis del crecimiento económico. En cambio, el concepto de difusión se ha removido del análisis económico, a menudo se resta importancia a la difusión ocupando un lugar secundario en el estudio. La difusión es según Antonelli,

...el núcleo del proceso de modernización. Se corresponde con fase en la que las innovaciones en proceso o productos han sido desarrolladas por un individuo o un grupo de innovadores, y las empresas o los consumidores están considerando la posibilidad de adoptarlas o no (1990:46).

El concepto de difusión tiene perspectiva multidisciplinaria dentro de la ciencia, aquí el fenómeno de difusión es percibido como el contagio y propagación de un virus en un espacio determinado, (figura 1). En este sentido, la importancia de estudiar la difusión radica en detectar factores que inciden en el proceso de contagio y precisar la relación entre estos factores de contagio en un entorno, $y$ al extrapolar el concepto de difusión al ámbito económico proyectar la idea de que una innovación tiene impacto económico en medida de la velocidad con cual se difunde.

\footnotetext{
${ }^{1}$ Grupo de investigadores europeo sobre los medios innovadores.

${ }^{2}$ Ver Olivier Crevoisier, (1994).
} 
Figura 1. Sistema Social

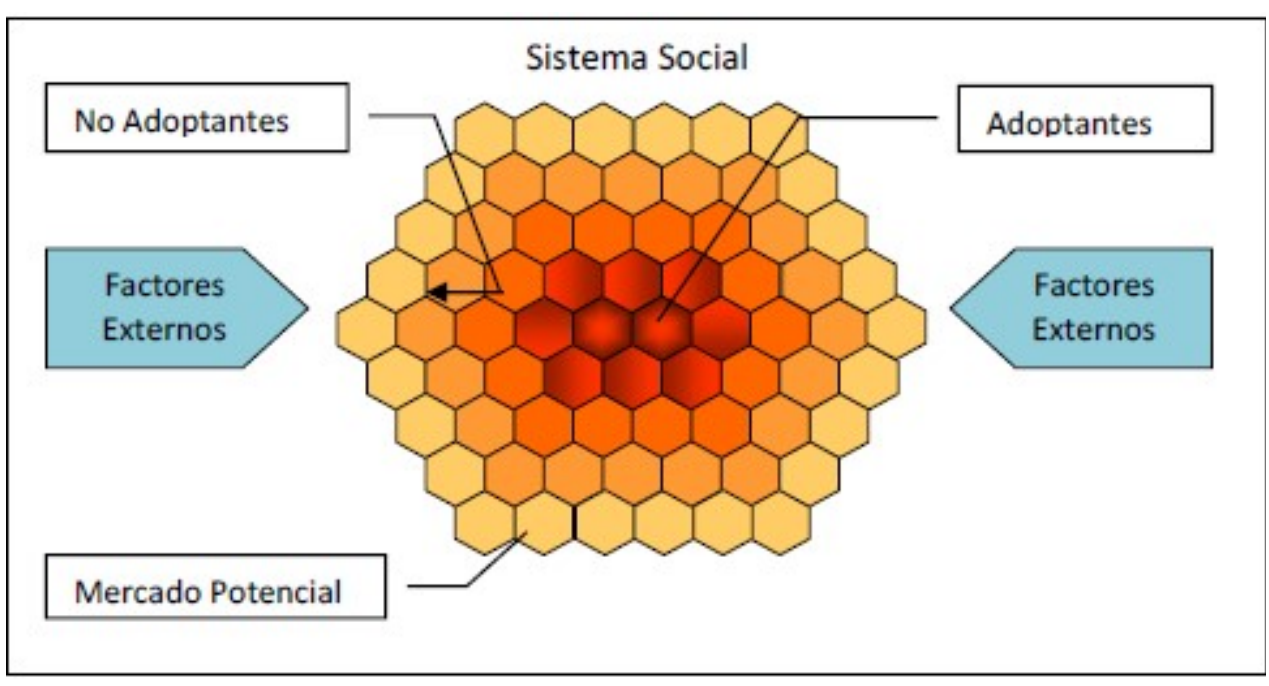

Fuente: elaborado por Herrera Madrid con datos de Antón, M. 1996.

Los modelos de difusión tecnológica se elaboran para analizar el nivel de propagación de una innovación o tecnología entre los miembros de un sistema social, que se representa por el total de potenciales adoptadores de la tecnología o innovación a través del tiempo (Mahajan y Muller, 1979).

Los primeros modelos de difusión tecnológica datan de la década de los setenta con las obras realizadas por Fourt y Woodlock, (1960) y Mansfield, (1961), años después Bass, (1969), fusiona los trabajos previos. En general las propuestas hacen referencia a la relación que existe entre los adoptantes y no adoptantes de un sistema social y el grado de abarrotamiento del mercado donde se introducía la innovación. La herramienta para calcular los parámetros en estos modelos con el procedimiento matemático de mínimos cuadrados ordinarios para hacer predicciones de la difusión de una innovación en el mercado.

La metodología que se pretende aplicar en el proyecto de tesis para determinar la difusión tecnológica de la industria aeroespacial en el sector secundario de la economía local es el modelo propuesto por Bass (1969). El modelo de Bass puede considerarse el más utilizado para explicar los procesos de difusión tecnológica.

Con el método seleccionado se estimarán los parámetros: el coeficiente de influencia externa: $p$, el coeficiente de influencia interna $q$ y el número total de potenciales adoptantes $A$, se realizará a través de la formulación discreta representada en la siguiente ecuación:

$$
x(t)=\bar{A}\left[\frac{1-e^{-(p+q) t}}{1+\left(\frac{q}{p}\right) e^{-(p+q) t}}-\frac{1-e^{-(p+q) t(-1)}}{1+\left(\frac{q}{p}\right) e^{-(p+q) t(-1)}}\right]+\varepsilon(\mathrm{t})
$$




\section{La industria aeroespacial: motor para el desarrollo}

La integración económica es resultado de la apertura de la economía mexicana y ha permitido conectar a México con grandes industrias. La industria aeroespacial es un ejemplo de esta conexión.

Las características tecnológicas que detenta la industria aeroespacial de manera general representan una fuente de innovación y tecnología para las regiones y localidades. Desde la perspectiva del desarrollo endógeno resalta la importancia de la rápida adopción de las innovaciones y tecnologías que permitan aumentar las capacidades internas de las regiones y así formar ventajas competitivas para acceder a procesos productivos globales. En este contexto, la constante interacción entre la industria aeroespacial y las regiones mexicanas no solo permiten el acceso a innovaciones y tecnologías que se emplean en el proceso productivo de la industria aeroespacial, sino que también la experiencia en el manejo de nuevos conocimientos en procesos y tecnología, aumenta la capacidad creativa e innovadora de los individuos dentro y fuera de la industria aeroespacial.

Para abordar la industria aeroespacial es necesario recurrir primeramente a una definición que refleje un panorama general de sus actividades para una mejor comprensión. La industria aeroespacial es definida por Gama Barranco, (2013: 2) como, “...la industria que se encarga del diseño, fabricación, comercialización y mantenimiento de aeronaves (aviones, helicópteros, vehículos aéreos, no tripulados, misiles, etc.,), y satélites espaciales”. La industria aeroespacial es origen de trabajos especializados, con funciones muy familiarizadas con las nuevas tecnologías, las cuales aportan condiciones que desencadenan la actividad innovadora. Los productos de la industria aeroespacial son el resultado de grandes inversiones en infraestructura, logística, capital humano y particularmente en investigación y desarrollo tecnológico.
A nivel global la inversión en la industria aeroespacial ha seguido una tendencia hacia las siguientes proporciones: “...algo más del $60 \%$ en producción, $18 \%$ en investigación y desarrollo y aproximadamente $20 \%$ en mantenimiento", (Universidad Politécnica de Madrid, 2011:38). Comparado con otros sectores de alta tecnología, la industria aeroespacial es líder en inversiones en investigación y desarrollo (Universidad Politécnica de Madrid, 2011:38).

La industria aeroespacial en México es reciente y dinámica, comenzó a principios de la década del 2000, pero particularmente a partir del año 2003 su crecimiento se ha profundizado alcanzando un promedio de 20\% anual; se coloca en el lugar número 15 entre los principales productores del mundo (Arbor, 2009:38). Esta cifra de crecimiento en la industria se ha traducido en un aumento en las exportaciones de la industria aeroespacial “...para el 2011, el monto de exportaciones mexicanas ascendió a 4,337 millones de dólares, logrando un saldo a favor de 555 mdd en la balanza comercial” (Secretaria de Economía, 2011). Por lo anterior, hoy en día la industria aeroespacial forma parte fundamental y estratégica de la agenda nacional, no solo por las inversiones que representa y el impacto positivo en el comercio, sino también por los efectos económicos que puede significar para las localidades y regiones: formación de mano de obra calificada y desarrollo tecnológico, (Hernández Chavira, 2013). México se convirtió en el principal receptor de inversiones destinadas a la manufactura. La estimación es que del año 1990 al 2009 las cifras han alcanzado los 33 mil millones de dólares (Pro-Aero 2012-2020. Programa Estratégico de la Industria Aeroespacial, 2011), (gráfica 1). En este panorama manufacturero México se coloca delante de países como China, y Rusia. 
Gráfica 1. Inversión de la industria aeroespacial en actividades manufactureras en México, 1990-2009

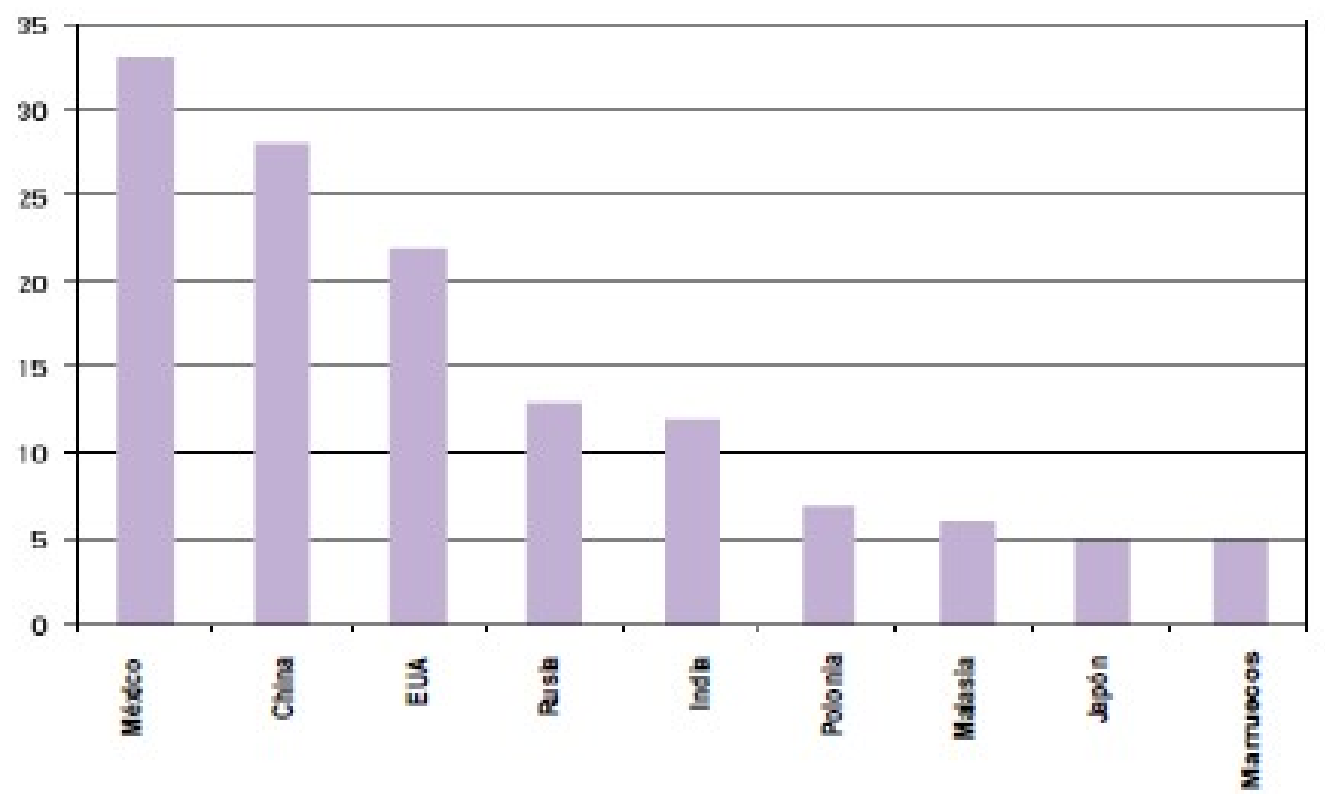

Fuente: Aerospace Globalization 2.0. AeroStrategy, November 2009.

La inversión de la industria aeroespacial al interior de México se distribuye de la siguiente manera, según Medina Ramírez, (2012): 79\% es para manufactura; 11\% para mantenimiento y reparación; y 10\% en investigación y desarrollo. A continuación se muestran la distribución porcentual en la gráfica 2.

Grafica 2. Distribucion de la inversion de la industria aeroespacial en Mexico

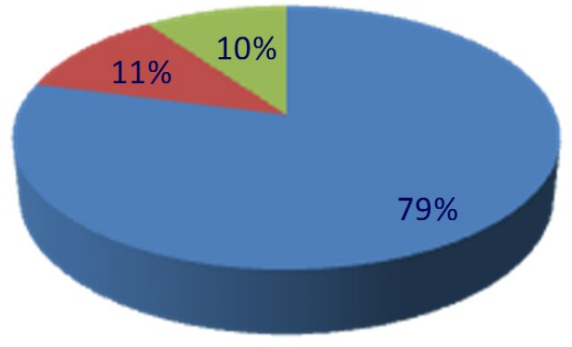

Manufactura

Mantenimiento y

reparación

Investigación y desarrollo

Fuente: elaboración propia a partir de la informacion de Medina Ramirez (2012).

En la tabla 1, se presenta la distribución de las empresas y los principales clúster de la industria aeroespacial en México. 
Tabla 1. Principales clúster de la industria aeroespacial en México

\begin{tabular}{|c|c|c|}
\hline PRINCIPALES CLUSTERS & ESPECIALIDAD & PRINCIPALES JUGADORES \\
\hline $\begin{array}{l}\text { En Baja California: } \\
\text { - Mexicali } \\
\text { - Tecate } \\
\text { - Tijuana }\end{array}$ & $\begin{array}{l}\text { Eléctrico-Electrónico } \\
\text { Manufactura de partes }\end{array}$ & $\begin{array}{l}\text { 51 empresas entre las que destacan: } \\
\text { - Honeywell } \\
\text { - Gulfstream Interiores Aéreos }\end{array}$ \\
\hline $\begin{array}{l}\text { En Chihuahua: } \\
\text { - Chihuahua } \\
\text { - Ciudad Juárez }\end{array}$ & $\begin{array}{l}\text { Manufactura de partes y } \\
\text { fuselajes, eléctrico- } \\
\text { electrónico, interiores, } \\
\text { mecanizados }\end{array}$ & $\begin{array}{l}28 \text { empresas entre las que destacan: } \\
\text { - Labinal, de Grupo Safran } \\
\text { - Cessna Aircraft } \\
\text { - Textron International } \\
\text { - Grupo American Industries } \\
\end{array}$ \\
\hline $\begin{array}{c}\text { En Querétaro: } \\
\text { - Querétaro }\end{array}$ & $\begin{array}{l}\text { Fabricación de componentes } \\
\text { de motor y trenes de } \\
\text { aterrizaje } \\
\text { Ensamble de componentes y } \\
\text { fuselajes de avión, } \\
\text { MRO }\end{array}$ & $\begin{array}{l}32 \text { empresas entre las que destacan: } \\
\text { - Bombardier } \\
\text { - ITP Ingeniería y Fabricación. } \\
\text { - SNECMA, de Grupo Safran } \\
\text { - Messier Dowty de Grupo Safran } \\
\text { - GE-IQ } \\
\text { - Aernnova }\end{array}$ \\
\hline $\begin{array}{l}\text { En Sonora: } \\
\text { - Hermosillo } \\
\text { - Guaymas } \\
\text { - Ciudad Obregón }\end{array}$ & $\begin{array}{l}\text { Manufactura de componentes } \\
\text { para motores y turbinas, } \\
\text { fuselaje y materiales } \\
\text { compuestos. }\end{array}$ & $\begin{array}{l}43 \text { empresas entre las que destacan: } \\
\text { - Goodrich Aerostructures de México } \\
\text { - ESCO }\end{array}$ \\
\hline $\begin{array}{l}\text { En Nuevo León: } \\
\text { - Apodaca } \\
\text { - Monterrey } \\
\text { - Santa Catarina }\end{array}$ & $\begin{array}{l}\text { Forjas y maquinados, } \\
\text { fabricación de componentes, } \\
\text { ensambles de fuselajes de } \\
\text { helicópteros. }\end{array}$ & $\begin{array}{l}29 \text { empresas entre las que destacan: } \\
\text { - Frisa Forjados } \\
\text { - MD Helicopters }\end{array}$ \\
\hline
\end{tabular}

Fuente: Secretaria de Economía. DGIPAT.

En Sonora se ubica el clúster aeroespacial mecanizado más importante e integral del país, las actividades que se realizan son el proceso de fundición, mecanizado y secundario. Particularmente el área de Guaymas, conformada por los municipios de Guaymas y Empalme es el hogar del clúster más importante de manufactura de componentes para turbina. Este sector se compone por 12 compañías, 10 de las cuales están situadas en el Parque Industrial Rocafuerte de Guaymas.

Con las ventajas competitivas de México, como son: posición geográfica y costos laborables bajos, se puede afirmar que prácticamente en Sonora se expresan las mismas ventajas competitivas. A continuación se presenta la tabla 2 que muestra la sincronía de las ventajas competitivas en la formación de clúster entre México y Sonora,

Tabla 2. Ventajas competitivas de México y Sonora

\begin{tabular}{|l|l|}
\hline Posición geográfica & Mano de obra calificada \\
\hline Costos de operación & Apoyo gubernamental \\
\hline Protección a la propiedad intelectual & Tratado de Libre Comercio de América del Norte \\
\hline Clústeres & Shelters (maquiladoras) \\
\hline
\end{tabular}

Fuente: Guerrero, 2013:124. 
Guerrero, (2013), contribuye de manera muy significativa al sintetizar cifras de la industria aeroespacial en Sonora. Lo anterior ayuda a dimensionar la importancia y la potencialidad que la industria aeroespacial representa para la entidad. La aportación que la industria aeroespacial hace al PIB sonorense es de 4\%, una cifra significativa teniendo en cuenta que se ha alcanzado en un lapso muy corto (tabla 3).

Tabla 3. Aportación de la industria aeroespacial al PIB de Sonora

\begin{tabular}{|l|l|}
\hline Aportación al PIB: $4.0 \%$ & $\begin{array}{l}\text { Fabricación: manufactura de } \\
\text { componentes de turbina, mantenimiento y } \\
\text { reparación de aeronaves, ensamble de } \\
\text { aeroestructuras, tratamientos términos y } \\
\text { procesos secundarios }\end{array}$ \\
\hline $\begin{array}{l}\text { Localización: Guaymas - Empalme, } \\
\text { Nogales, Hermosillo y Cd. Obregón }\end{array}$ & Número total de empresas: 50 empresas \\
\hline Empleos directos: 9,000 empleos & Empleos indirectos: 4,000 empleos \\
\hline $\begin{array}{l}\text { Inversiones concretadas 2009 - 2013: 15 } \\
\text { proyectos aeroespaciales (7 en expansión } \\
\text { y } 8 \text { nuevas inversiones) }\end{array}$ & $\begin{array}{l}\text { Inversión extranjera directa (IED) 2009 - } \\
\mathbf{2 0 1 3}: 128.43 \text { millones de dólares }\end{array}$ \\
\hline
\end{tabular}

Fuente: Guerrero, (2013:126).

La estrategia del Estado de Sonora se basa en el desarrollo de la cadena de proveeduría con un enfoque de innovación principalmente en la fabricación de turbinas, así como la generación del talento especializado en el tema con el enfoque en las necesidades de la industria aeroespacial. El Estado planea continuar las estrategias de corto, mediano y largo plazo que le permitan ser un líder mundial en la manufactura de turbinas, las acciones para lograr esto se encuentra en costos competitivos en toda la cadena de producción, el desarrollo de talento y un desarrollo de los administradores locales. ${ }^{1}$

\section{Conclusiones}

En este trabajo se presentaron distintas teorías que proporcionan herramientas para analizar e interpretar la integración económica del sistema productivo local a la industria aeroespacial. Además se proporcionan elementos teóricos para dimensionar la industria aeroespacial desde una perspectiva del desarrollo endógeno que dé pie al desarrollo de las capacidades internas de la región y la localidad.

Las cifras que describen a la industria aeroespacial en México nos muestran que se ha registrado un importante crecimiento principalmente en la última década. Como se mencionó, el crecimiento observado por la industria aeroespacial tiene diferentes explicaciones relacionadas con los bajos costos de producción, la experiencia mexicana en la industria automotriz y electrónica aportan factores que favorecen el desarrollo de la industria aeroespacial en el territorio nacional.

En el caso de Sonora se puede asegurar que es la representación arquetipo de la industria aeroespacial en México. El comportamiento nacional de la industria aeroespacial es muy similar al comportamiento que presenta la industria en Sonora. Por lo anterior, Sonora debe crear las condiciones a través de estrategias que permitan mantener y acrecentar la relación económica que existe con la industria aeroespacial.

\footnotetext{
${ }^{1}$ http://www.promexico.gob.mx/es/mx/aeroespacial-inversion
} 


\section{Referencias:}

Antonelli, C. (1990). La difusión internacional de innovaciones: pautas, determinantes y efectos. Pensamiento iberoamericano. No. 16, Pp. 45-56.

Arbor, A. (2009). Aerospace Globalization 2.0: Implications for Canada's Aerospace Industry. November. BANCOMEXT. (2010). La visión estratégica del Bancomext. Diciembre. http://www.revistacomercioexterior.com/noticias/print. php?story_id=206

Becerra, A. (2013). Integración de la economía de Sonora a la industria aeroespacial. Fundamentos teóricos y de contexto. Tendencias económicas. Año 4, vol. 7, enero-junio. Pp. 117-134.

Balassa, Bela (1962). The Theory of Economic Integration. Londres. George Allen and Unwin.

Bass, F. M. (1969). A new product growth model for consumer durables. Management Science, 15(1). Pp. 215-227.

Becattini G. (1992). Distretti industriali. Enciclopedia dell'economia. Garzanti, Milano.

Cheriet F. (2010). Performance et dynamiques des réseaux d'alliances stratégiques asymétriques, Working Paper Moisa.

Conesa, E. R. (1982). Conceptos fundamentales de integración económica. Integración latinoamericana 71. Pp. 2-27.

Crevoisier, O. (1994). Dynamique industrielle et dynamique régionale: l'articulation par les milieux innovateurs, revue d'économie industrielle. Vol. 70, pp 33-48).

Dupuigrenet-Desroussilles Guy. (1964). Balassa (Bela) The theory of economic integration. In: Revue économique. Volume15, n¹. Pp. 145-146.

Fourt, L. y Woodlock, J. (1960). Early prediction of market success for grocery products. Journal of marketing. Vol. 25, Octubre. Pp. 31-38.
Hernández, J. (2010). Mecanismos de aprendizaje en la transferencia de conocimiento de los componentes estructurales del modelo Q400 y global express: el caso de bombardier aeroespacial, Querétaro. Universidad autónoma metropolitana.

Machlup, F. (1977). A History of Thought on Economic Integration. New York. Columbia. University Press.

Madhavan (2001). Cooperative networks and competitive dynamics: a structural embeddedness perspective. The academy of management review. Vol. 26, No. 3, pp 431-445.

Mahajan y Muller (1979). Innovation diffusion and new product growth models in marketing. Journal of marketing, 43, 4. Pp. 55-68.

Mansfield, E. (1961). Technological change and rate of imitation. Econometrica. Vol. 29. Pp. 741-766.

Medina Ramírez, S. (2012). El despegue de la industria aeroespacial en México. Comercio Exterior. Vol. 62, No. 6. Pp. 1-9.

Perroux, F. (1967). ¿Quién integra? ¿En beneficio de quién se realiza a integración? Integración y comercio 1. Pp. 33-53.

Promexico.

http://www.promexico.gob.mx/es/mx/aeroespacialinversion

Secretaria de Economía. (2011). Pro-Aero 2012-2020. Programa Estratégico de la Industria Aeroespacial. FEMIA.

Tremblay, Diane-Gabrielle (2006). Réseaux, clusters, communautés de pratique et développement des connaissances. Chaire Bell en technologies et organisation du travail. Université du Québec.

Vázquez Barquero, A. (1999). Desarrollo, redes e innovación: lecciones sobre desarrollo endógeno. Pirámide, Madrid.

Vázquez Barquero, A. (2005). Las nuevas fuerzas del desarrollo. Antoni Bosch Editor. Madrid, España. 
Viner, J. (1950). Teoría de las uniones aduaneras. Una aproximación al libre comercio. En Viner. The customs union issue. Pp. 89-103. New York. Carnegie Endowment for International Peace. 